\title{
Treatment with Anlotinib After Chemotherapy and EGFR-TKI Resistance in Lung Adenosquamous Carcinoma with Concurrent EGFR and PIK3CA Mutations: A Case Report and Literature Review
}

\author{
Yonghui $\mathrm{Wu}\left(\mathbb{D}^{1, *}\right.$ \\ Kai Zhang ${ }^{1, *}$ \\ Jiexia Guan ${ }^{2}$ \\ Weibin $\mathrm{Wu}^{\prime}$ \\ Jian Zhang' \\ Huiguo Chen' \\ 'Department of Cardiothoracic Surgery, \\ The Third Affiliated Hospital of Sun Yat- \\ Sen University, Guangzhou, Guangdong, \\ 510630, People's Republic of China; \\ ${ }^{2}$ Department of Pathology, The Third \\ Affiliated Hospital of Sun Yat-Sen \\ University, Guangzhou, Guangdong, \\ 510630, People's Republic of China \\ *These authors contributed equally to \\ this work
}

\begin{abstract}
Concurrent mutations of epidermal growth factor receptor (EGFR) and phosphatidylinositol-4,5-bisphosphate 3-kinase catalytic subunit alpha (PIK3CA) in non-small cell lung cancer (NSCLC) are rare, and the presence of concurrent mutations may complicate treatment. Herein, we report a case of primary lung adenosquamous carcinoma with concurrent EGFR 21 (L858R) and PIK3CA (H1047R/E545K) mutations, and the results of a literature review to help management and treatment. A 49-year-old female was admitted our department for coughing and excessive sputum production for more than 1 month. Computed tomography (CT) of the chest identified a lesion, and a CT-guided needle biopsy was performed. Pathological examination and immunohistochemistry (IHC) staining confirmed a diagnosis of primary lung adenosquamous carcinoma. Amplification refractory mutation system-polymerase chain reaction (ARMS-PCR) gene sequencing demonstrated mutations in both EGFR 21 (L858R) and PIK3CA (H1047R/E545K) mutations in adenocarcinoma (AC) component. She was treated with pemetrexed plus platinum-based chemotherapy and an EGFR-tyrosine kinase inhibitor (TKI). Disease progression occurred with gefitinib or osimertinib as maintenance therapy. A repeat CT-guided needle biopsy was performed, and generation sequencing (NGS) revealed EGFR 21 (L858R) and PIK3CA (H1047R/E545K) mutations. Anlotinib monotherapy was then administered as the thirdline treatment, and there was a PR. The patient is currently still receiving treatment and follow-up. To our knowledge, there is little evidence that anlotinib is beneficial when there are concurrent EGFR and PIK3CA mutations. PIK3CA mutations are associated with poor therapeutic effects and short survival time. Concurrent EGFR and PIK3CA mutations do not respond to EGFR-TKI treatment. Chemotherapy should be given in combination with a TKI and can prolong the progression-free survival (PFS) and overall survival (OS) of patients with lung cancer.
\end{abstract}

Keywords: EGFR mutation, PIK3CA mutation, resistant mutation, adenosquamous carcinoma, anlotinib

\section{Introduction}

Lung cancer is the leading cause of cancer death in China, and around the world. Patients with advanced non-small cell lung cancer (NSCLC) patients are not surgical candidates, and while chemotherapy is the major treatment the results are disappointing. Over the last decade, targeted therapy has become an important treatment for patients with EGFR mutations, ALK mutations, and other sensitive
Department of Cardiothoracic Surgery, The Third Affiliated Hospital of Sun YatSen University, Tianhe Road NO. 600, Guangzhou, Guangdong, 510630, People's Republic of China

Email Andychen820727@hotmail.com 
mutations. ${ }^{1}$ Exon 19 del and 21 (L858R) mutations in EGFR are the most common sensitive mutations, and T790M and 20 exon insertion are the common resistant mutations. $^{2}$ There are also other mutations that can occur concurrently such as those in KRAS, PIK3CA, and PTEN which can benefit or hinder treatment.

The PI3K family is involved in a number of normal cellular processes. PI3K can activate phosphorylation of AKT and downstream signal transduction pathways, such as the PI3K-AKT-mTOR pathway, which plays a central role in regulating tumor cell growth, reproduction, migration, and apoptosis. However, the role of PIK3CA mutations in NSCLC is still being debated. Some studies indicate that $P I K 3 C A$ mutations are an independent risk factor for overall survival (OS) and progression-free survival (PFS) in patients with NSCLC. On the other hand, Song et al and Zhang et al showed that PIK3CA mutations do not significantly affect the survival time of patients with NSCLC. ${ }^{3,4}$

PIK3CA mutations often occur concurrently with EGFR mutations. However, few studies have examined the effect of PIK3CA mutations on outcomes in patients with lung cancer. Herein, we report a case of primary lung adenosquamous carcinoma with concurrent EGFR 21 (L858R) and PIK3CA (H1047R/E545K) mutations, and the results of a literature review to help management and treatment.

\section{Case Report}

A 49-year-old female was admitted to our department with a chief complaint of coughing and excessive sputum production for more than 1 month. Chest computed tomography (CT) demonstrated a lesion located in the upper lobe of right lung, and multiple nodules scattered in the bilateral lungs, consistent with a diagnosis of primary lung cancer and metastases (Figure 1A1-A3).

She was previously healthy, had no significant medical history, and there was no history of cancer history or any other disease in her family. She was a non-smoker and not exposed to second-hand smoke. Neck and supraclavicular lymph nodes were not enlarged and not palpable on physical examination.

Laboratory examinations showed that blood carcinoembryonic antigen (CEA), neuron-specific enolase (NSE), and cytokeratin 19 fragment (CTFRA21-1) levels were normal. Positron emission tomography-CT (PET-CT) and brain magnetic resonance imaging (MRI) scans showed metastases in the bilateral lungs, and no metastases in the liver, brain, adrenal glands, bone, or other organs. A CT-guided needle biopsy was performed of the right upper lobe lesion. Histopathological examination and immunohistochemical (IHC) staining were consistent with a diagnosis of primary lung adenosquamous carcinoma. Staining for cytokeratin 5/6 (CK5/6), P40, thyroid transcription factor-1 (TTF-1), and Napsin-A was positive. The Ki-67 index was about $70 \%$ in the squamous cell carcinoma (SCC) component, and about 30\% in the adenocarcinoma (AC) component (Figure 2A-F). ARMSPCR sequencing demonstrated concurrent EGFR exon 21 (L858R) and PIK3CA (H1047R/E545K) mutations in the AC component (Figure 3A-E) She was diagnosed with primary lung adenosquamous carcinoma and bilateral lung metastases, disease stage IVA.

She was treated with pemetrexed $\left(500 \mathrm{mg} / \mathrm{m}^{2}\right)$ plus platinum-based chemotherapy every 3-4 weeks, and gefitinib $250 \mathrm{mg}$, orally, once daily. After 2 cycles, efficacy evaluation indicated a partial response (PR) (Figure 1B). After another 2 cycles of the same therapy evaluation indicated steady disease (SD) (Figure 1C). She was then begun on gefitinib alone as maintenance therapy. After 2 months, she developed an intermittent cough and hemoptysis, chest CT illustrated that the tumor and metastases had increased in size (Figure 1D).

The patient declined a repeat biopsy for nextgeneration sequencing (NGS), and she was begun on the same chemotherapy regime and osimertinib $80 \mathrm{mg}$, orally, once daily. After 4 cycles, evaluation indicated a PR (Figure 1E). Adverse events included a grade 1/2 rash and anemia, and grade $1 / 2$ hematological toxicity (decreased white blood cells and platelets). After osimertinib only for maintenance therapy for 2 months, she developed an intermittent cough again and evaluation indicated progressive disease (PD) (Figure 1F).

The time from diagnosis to the second occurrence of PD was about 10 months. A repeat CT-guided needle biopsy was performed, and pathological examination was consistent with adenosquamous carcinoma. The frequency of SCC and AC components in the tumor, and IHC staining results and the Ki-67 index were almost the same as the first evaluations. NGS demonstrated concurrent EGFR 21 (L858R) and PIK3CA (H1047R/E545K) mutations. No $T 790 M$, C797S, or other mutations were identified.

The patient declined further chemotherapy and was treated with anlotinib as the third-line regime (a dose of $12 \mathrm{mg} /$ day for 2 weeks, stop for 1 week, and then repeat). After 2 cycles, evaluation indicated a PR (Figure 1G), and 

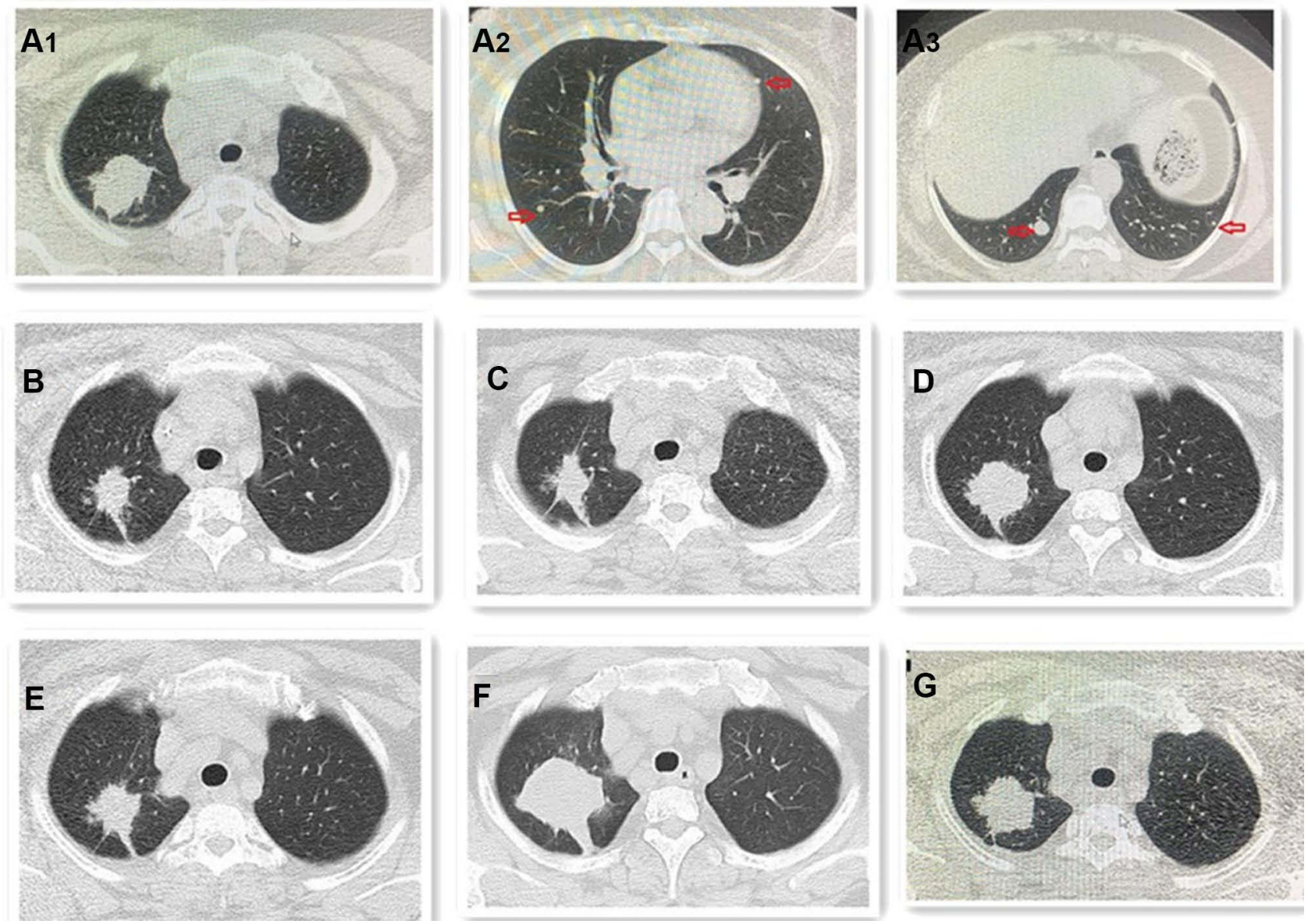

Figure I The chest CT outcomes during the whole treatment. (A) (AI-A3) Chest CT reveals primary lung cancer and metastases (red arrow); (B) tumor was PR after 2 cycles chemotherapy and gefitinib; (C) tumor was SD after 2 cycles chemotherapy and gefitinib again; (D) tumor was PD for taking gefitinib only; (E) tumor was PR again after 4 cycles the same chemotherapy and osimertinib; (F) tumor was PD again for taking osimertinib only. (G) Tumor was PR again after 2 cycles anlotinib.

SD after 4 cycles. The only adverse effect was grade 1 hand-foot syndrome. She continues to receive treatment and follow-up.

\section{Discussion}

Surgery, chemotherapy, and radiotherapy are the traditional treatments for NSCLC; however, the development of molecular targeted therapies and immune therapies have improved outcomes and are currently important methods of treatment. EGFR mutations and ALK fusion are sensitive to molecular targeted inhibitors, which can prolong the PFS and OS of patients. ${ }^{5}$ Patients harboring EGFR mutations benefit from treatment with EGFR-TKIs, and the median PFS (mPFS) with this treatment is 10-14 months. In order to improve EGFR-TKI efficacy and overcome acquired resistance, an EGFR-TKI plus chemotherapy are widely used in patients with advanced NSCLC harboring mutations. ${ }^{6}$ Han et $\mathrm{al}^{7}$ showed that the PFS of patients treated with an EGFR-TKI and chemotherapy was
17.5 months, which was longer than that of chemotherapy alone (5.7 months) and gefitinib alone (11.9 months). The objective response rates (ORR) in the combination group, chemotherapy group, and gefitinib group were $82.5 \%$, $32.5 \%$, and $65.9 \%$, respectively. The OS of the combined group was significantly prolonged without a significant increase in adverse events.

The occurrence and development of lung cancer is a complicated process involving many signaling molecules and pathways. EGFR, ALK, Met, Her-2, KRAS, Ros-1, et al, which involve in the sensitive mutations and resistant mutations. Mutations causing resistance to treatment have brought many obstacles for therapy. ${ }^{8}$ The PI3K-AKT-mTOR signaling pathway has received a large amount of attention in NSCLC. PI3K plays an important role in regulating cell proliferation, inhibiting apoptosis, and promoting tumor angiogenesis and tumor invasion. ${ }^{9}$ PIK3CA mutations are the only tumor-specific mutations in the PI3K family, which mainly occur in 

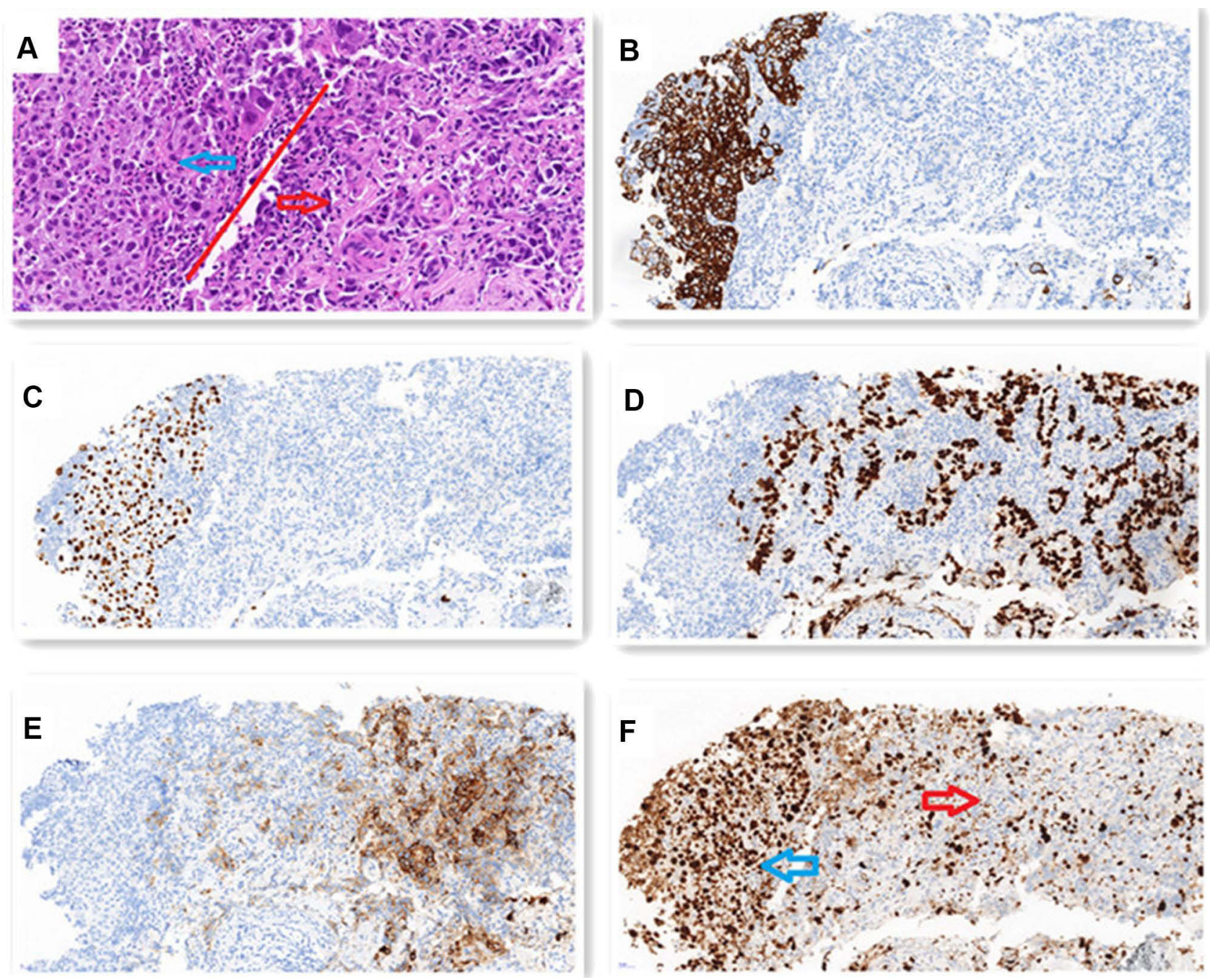

Figure 2 (A) H-E staining shows that SCC (blue arrow) presents a solid sheet-like arrangement. The cell cytoplasm is red stained, the nucleus is large, nucleoli and mitosis, no intracellular keratinizing and keratinizing beads are seen, but AC (red arrow) is adenoid, cord-like or solid arrangement. The cell cytoplasm is reddish or translucent, the nucleus is large and irregular, and nucleoli and mitosis can be seen. ( $\times 200)$. SCC accounts for about $10 \%$ and AC accounts for about $90 \%$ of the tumor. IHC staining shows: (B) CK5/6 and (C) P40 are positive in SCC components. (x200) (D) TTF-I and (E) Napsin-A are positive in AC components. (x200) (F) Ki-67 index is about 70\% in SCC (blue arrow) and about $30 \%$ in AC (red arrow). (x200).

Exon 9 and Exon 20. Mutations have been regarded as a co-occurring mutations rather than the main mutations, but concurrent mutations affect clinical outcomes in patients with lung cancer. ${ }^{10}$

The PIK3CA mutation rate in NSCLC is $2-5 \%, 8-10 \%$ in lung squamous cell carcinoma, and $2.8 \%$ in adenocarcinoma. ${ }^{11}$ PIK3CA inhibitors have been studied and used for treatment of patients with lung cancer. Corey et al treated 21 patients who failed first-line platinum combined chemotherapy with taselisib, and the mPFS and OS were 2.9 months and 5.3 months, respectively. ${ }^{12}$ Van et al treated patients with NSCLC and PIK3CA mutations with buparlisib, and the lung cancer group compared with non-squamous lung cancer group, the patient's 12-week PFS rate was $23.3 \%$ versus $20.1 \%$, respectively. The anti-tumor activity of a single PIK3CA inhibitor may be limit, and combined therapy may be more effective than treatment with a single inhibitor. ${ }^{13}$ Zhou Xin et al demonstrated that LY294002, an inhibitor of PI3K, was able to increase the sensitivity of erlotinib in NSCLC cells in vitro. ${ }^{14}$

Immunotherapy has also been used to treat patients with PIK3CA mutations, but the PIK3/AKT pathway has been reported to be associated with immunotherapy resistance. ${ }^{15}$ Diego et $\mathrm{al}^{16}$ treated patients with PIK3CA mutations with nivolumab; however, all patients did not respond. It was speculated that PIK3CA mutations predict a poor response to immunotherapy.

Concurrent EGFR and PIK3CA mutations are rare in lung cancer, ${ }^{12}$ which has been found in approximately 


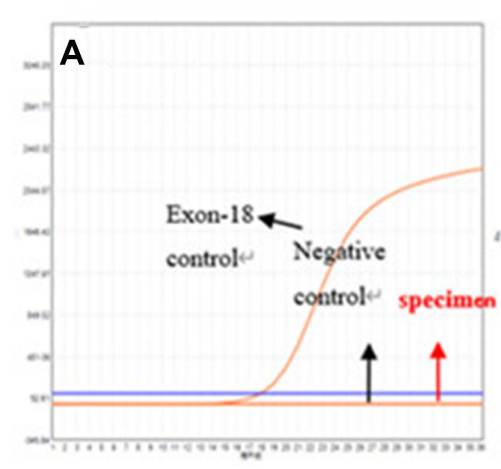

EGFR geneExon-18

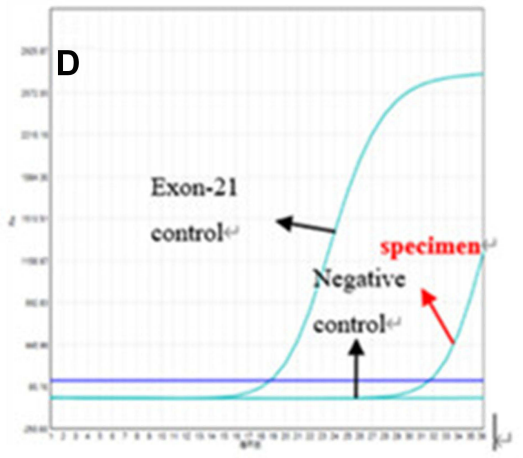

EGFR gene Exon-21

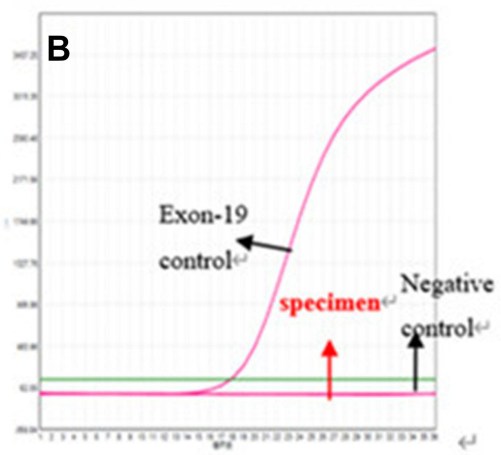

EGFR gene Exon-19

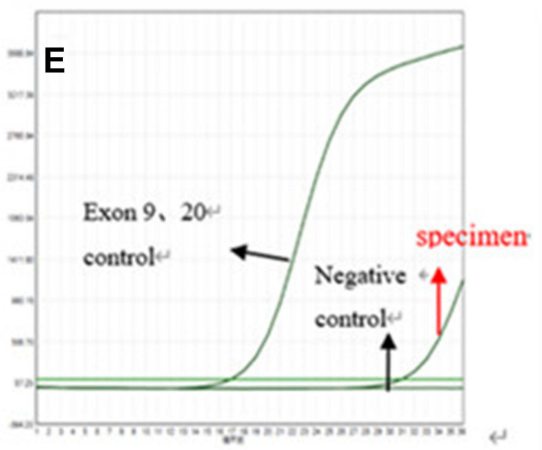

PIK3CA gene Exon-9、 20

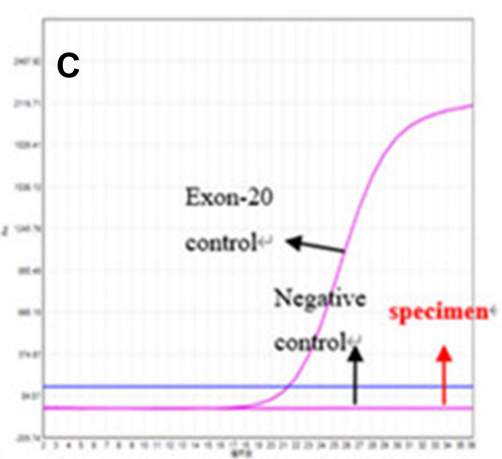

EGFR gene Exon-20

Figure 3 ARMS-PCR gene sequencing illustrated EGFR 18 (A), EGFR 19 (B), EGFR 20 (C) are negative, EGFR 21 (L858R) (D) and PIK3CA (HI047R/E545K) (E) concurrent mutations in $A C$ component.

$3.5 \%$ of EGFR mutation patients. ${ }^{17}$ The presence of coexisting mutations is considered to be associated with a poor response to treatment and short survival time. ${ }^{18}$ Wang et al showed that PIK3CA mutations are related to OS and PFS, ${ }^{3}$ affect EGFR-TKI sensitivity, and have a poor prognosis. Chang et $\mathrm{al}^{19}$ reported that patients treated with a EGFG-TKI without RAS/PIK3CA/PTEN mutations had a mPFS of 15.1 months and OS of 53.8 months, but for patients with RAS/PIK3CA/PTEN mutations the mPFS was only 19.9 months and OS was only 27.4 months.

There are limited data on the use of vascular endothelial growth factor-TKIs (VEGF-TKIs) to overcome treatment resistance due to EGFR and PIK3CA mutations. Yang et al reported that treatment with a combination of apatinib and osimertinib can overcome resistance due to $P I K 3 C A$ mutation. ${ }^{20} \mathrm{Yu}$ et al reported the case of a patient with a pulmonary artery sarcoma with $P I K 3 C A$ mutations; the patient was treated with chemotherapy followed by anlotinib and the survival time was 8 months. ${ }^{21}$ Jonasch $E$ et al reported that VEGF inhibitor showed a similar efficacy to the mTOR inhibitor everolimus. ${ }^{22}$ Anlotinib is a new oral TKI that targets the VEGF receptor, fibroblast growth factor receptor (FGFR), platelet-derived growth factor receptor (PDGFR), and c-kit. ${ }^{23}$ It is recommended as a third-line treatment in patients with lung cancer. However, there is currently no literature regarding using anlotinib to treat lung cancer patients with EGFR and PIK3CA mutations.

In our case, there was tumor and metastasis progression when the patient was on gefitinib maintenance or osimertinib maintenance treatment. Shen et al demonstrated that chemotherapy plays an important role in prolonging OS of patients with EGFR mutations combined with KRAS, PIK3CA, or HER-2 mutations. ${ }^{24}$ Chemotherapy and a TKI should be used in combination. $^{25}$ But for the patient who cannot tolerate chemotherapy, anlotinib may be an alternative that can control the primary tumor and metastases.

The primary limitation of this report is that long-term follow-up is needed to evaluate the long-term effects of anlotinib. 


\section{Conclusion}

Concurrent EGFR and PIK3CA mutations are rare in NSCLC. PIK3CA is mostly a co-occurring mutation and is inherently resistant to EGFR-TKIs and predicts a poor prognosis. The effects of EGFR-TKI or PIK3CA inhibitor alone are not beneficial for patients, and perhaps even immunotherapy is not effective for PIK3CA mutation. An EGFR-TKI combined with chemotherapy can prolong PFS and OS in patients with lung cancer. Anlotinib may be an alternative that can control the primary tumor and metastases.

\section{Abbreviations}

EGFR, epidermal growth factor receptor; PIK3CA, phosphatidylinositol-4,5-bisphosphate 3-kinase catalytic subunit alpha; NSCLC, non-small cell lung cancer; CT, computed tomography; IHC, immunohistochemistry; ARMS-PCR, amplification refractory mutation system polymerase chain reaction; AC, adenocarcinoma; TKI, tyrosine kinase inhibitor; PR, partial response; PD, progression disease; NGS, next-generation sequencing; PFS, progression-free survival; OS, overall survival; PET-CT, positron emission tomography-CT; MRI, brain magnetic resonance imaging; SCC, squamous cell carcinoma; SD, steady disease; mPFS, median PFS; ORR, objective response rate.

\section{Data Sharing Statement}

For patients' privacy, the patient information is publicly inaccessible.

\section{Ethics Approval and Consent to Participate}

This study was approved by institutional ethics committee of Third Affiliated Hospital of Sun Yat-Sen University, Guangzhou, China. Written informed consent to participate was obtained from the patient.

\section{Consent for Publication}

Written informed consent for publication has been obtained from the patient stating that the details/images can be available on the Internet and may be seen by the general public.

\section{Acknowledgments}

We would like to thank the patient and her family for giving consent for publication.

\section{Author Contributions}

All authors made substantial contributions to conception and design, acquisition of data, or analysis and interpretation of data; took part in drafting the article or revising it critically for important intellectual content; agreed to submit to the current journal; gave final approval of the version to be published; and agreed to be accountable for all aspects of the work.

\section{Funding}

There is no funding to report.

\section{Disclosure}

The authors have no competing interests to declare.

\section{References}

1. Suh JH, Johnson A, Albacker L, et al. Comprehensive genomic profiling facilitates implementation of the national comprehensive cancer network guidelines for lung cancer biomarker testing and identifies patients who may benefit from enrollment in mechanismdriven clinical trials. Oncologist. 2016;21(6):684-691. doi:10.1016/j. jtho.2016.11.1818

2. Murtuza A, Bulbul A, Shen JP, et al. Novel third-generation EGFR tyrosine kinase inhibitors and strategies to overcome therapeutic resistance in lung cancer. Cancer Res. 2019;79(4):689-698. doi:10. 1158/0008-5472.can-18-1281

3. Wang Y, Wang Y, Li J, Li J, Che G. Clinical significance of PIK3CA gene in non-small-cell lung cancer: a systematic review and meta-analysis. Biomed Res Int. 2020;2020:1-9. doi:10.1155/2020/ 3608241

4. Song Z, Yu X, Zhang Y. Mutation and prognostic analyses of PIK3CA in patients with completely resected lung adenocarcinoma. Cancer Med. 2016;5(10):2694-2700.doi. doi:10.1002/cam4.852

5. Jin $\mathrm{Y}$, Bao $\mathrm{H}$, Le $\mathrm{X}$, et al. Distinct co-acquired alterations and genomic evolution during TKI treatment in non-small-cell lung cancer patients with or without acquired T790M mutation. Oncogene. 2019;39(9):1846-1859. doi:10.1038/s41388-019-1104-z

6. Rebuzzi SE, Alfieri R, La Monica S, Minari R, Petronini PG, Tiseo M. Combination of EGFR-TKIs and chemotherapy in advanced EGFR mutated NSCLC: review of the literature and future perspectives. Crit Rev Oncol Hematol. 2020;146:102820. doi:10. 1016/j.critrevonc. 2019.102820

7. Han B, Jin B, Chu T, et al. Combination of chemotherapy and gefitinib as first-line treatment for patients with advanced lung adenocarcinoma and sensitive EGFR mutations: a randomized controlled trial. Int J Cancer. 2017;141(6):1249-1256. doi:10.1002/ ijc. 30806

8. Amos C, Tsongalis G, Deharvengt S, et al. Identifying associations between somatic mutations and clinicopathologic findings in lung cancer pathology reports. Methods Inf Med. 2018;57(01/02):63-73. doi:10.3414/me17-01-0039

9. Milano G. PIK3CA mutations and specific treatment: do not forget lessons from RAS mutations and EGFR targeting. Cancer Chemother Pharmacol. 2020;85(2):473-474. doi:10.1007/s00280-019-04016-9

10. Blakely $\mathrm{CM}$, Watkins $\mathrm{TBK}, \mathrm{Wu} \mathrm{W}$, et al. Evolution and clinical impact of co-occurring genetic alterations in advanced-stage EGFR-mutant lung cancers. Nat Genet. 2017;49(12):1693-1704.doi. doi:10.1038/ng.3990 
11. Aredo JV, Padda SK, Kunder CA, et al. Impact of KRAS mutation subtype and concurrent pathogenic mutations on non-small cell lung cancer outcomes. Lung Cancer. 2019;133:144-150. doi:10.1016/j. lungcan.2019.05.015

12. Langer CJ, Redman MW, Wade JL, et al. SWOG S1400B (NCT02785913), a Phase II Study of GDC-0032 (Taselisib) for Previously Treated PI3K-Positive Patients with Stage IV Squamous Cell Lung Cancer (Lung-MAP Sub-Study). $J$ Thoracic Oncol. 2019;14(10):1839-1846. doi:10.1016/j.jtho.20 19.05 .029

13. Vansteenkiste JF, Canon JL, De Braud F, et al. Safety and Efficacy of Buparlisib (BKM120) in Patients with PI3K Pathway-Activated Non-Small Cell Lung Cancer: results from the Phase II BASALT-1 Study. J Thorac Oncol. 2015;10(9):1319-1327. doi:10.1097/JTO.00 00000000000607

14. Zhou X, Wang X, Zhu H, et al. PI3K inhibition sensitizes EGFR wild-type NSCLC cell lines to erlotinib chemotherapy. Exp Ther Med. 2021;21(1):9. doi:10.3892/etm.2020.9441

15. Borcoman E, De La Rochere P, Richer W, et al. Inhibition of PI3K pathway increases immune infiltrate in muscle-invasive bladder cancer. Oncoimmunology. 2019;8(5):e1581556. doi:10.1080/21624 02X.2019.1581556

16. Kauffmann-Guerrero D, Tufman A, Kahnert K, et al. Response to checkpoint inhibition in non-small cell lung cancer with molecular driver alterations. Oncol Res Treatment. 2020;43(6):289-298. doi:10. $1159 / 000506842$

17. Guo YJ, Song J, Wang Y, et al. Concurrent genetic alterations and other biomarkers predict treatment efficacy of EGFR-TKIs in EGFR-mutant non-small cell lung cancer: a review. Front Oncol. 2020:10. doi:10.3389/fonc.2020.610923.
18. Vanderlaan P, Rangachari D, Mockus S, et al. P1.02-031 Mutations in TP53, PIK3CA, PTEN and other genes in EGFR mutated lung cancers: correlation with clinical outcomes. J Thoracic Oncol. 2017;12(1):S506. doi:10.1016/j.jtho.2016.11.614

19. Chang WJ, Sung JS, Lee SY, et al. The clinical significance of RAS, PIK3CA, and PTEN mutations in non-small cell lung cancer using cell-free DNA. J Clin Med. 2020;9(8):2642. doi:10.3390/jcm9082642

20. Yang X, Xia Y, Xu L, et al. Efficacy and safety of combination treatment with apatinib and osimertinib after osimertinib resistance in epidermal growth factor receptor-mutant non-small cell lung carcinoma-A retrospective analysis of a multicenter clinical study. Front Mol Biosci. 2021;8:639892.doi. doi:10.3389/fmolb.2021.639892

21. Wu Y, Huang J, Wang Q, et al. Whole-exome sequencing insights into pulmonary artery sarcoma mimicking pulmonary embolism: a case report and review. Onco Targets Ther. 2019;12:6227-6235. doi. doi:10.2147/OTT.S212416

22. Jonasch E, Hasanov E, Corn PG, et al. A randomized Phase 2 study of MK-2206 versus everolimus in refractory renal cell carcinoma. Ann Oncol. 2017;28(4):804-808. doi:10.1093/annonc/mdw676

23. Chi Y, Fang Z, Hong X, et al. Safety and efficacy of anlotinib, a multikinase angiogenesis inhibitor, in patients with refractory metastatic soft-tissue sarcoma. Clin Cancer Res. 2018;24(21):5233-5238. doi:10.1158/1078-0432.CCR-17-3766

24. Shen HB, Li J, Yao YS, et al. Impact of somatic mutations in non-smallcell lung cancer: a retrospective study of a Chinese cohort. Cancer Manag Res. 2020;12:7427-7437. doi:10.2147/cmar.s254139

25. Lei L, Wang W, Zhu Y, et al. Potential mechanism of primary resistance to icotinib in patients with advanced non-small cell lung cancer harboring uncommon mutant epidermal growth factor receptor: a multi-center study. Cancer Sci. 2020;111(2):679-686. doi:10.1111/cas.14277

\section{Publish your work in this journal}

Cancer Management and Research is an international, peer-reviewed open access journal focusing on cancer research and the optimal use of preventative and integrated treatment interventions to achieve improved outcomes, enhanced survival and quality of life for the cancer patient.
The manuscript management system is completely online and includes a very quick and fair peer-review system, which is all easy to use. Visit http://www.dovepress.com/testimonials.php to read real quotes from published authors. 